\title{
ThE GIANT COMPONENT AFTER PERCOLATION OF PRODUCT GRAPHS
}

\author{
Lyuben Lichev \\ Ecole Normale Supérieure de Lyon, Lyon, France
}

October 19, 2021

\begin{abstract}
In this paper we show the existence of a sharp threshold for the appearance of a giant component after percolation of Cartesian products of graphs under assumptions on their maximum degrees and their isoperimetric constants. In particular, this generalises a work of Ajtai, Komlós and Szemerédi from 1982 concerning percolation of the hypercube in high dimension.
\end{abstract}

Keywords: giant component, product graph, percolation, random graph, sharp threshold MSC Class: 05C76, 05C80

\section{Introduction}

The field of random graphs was born in a series of papers of Erdôs and Rényi [13, 14, 15. The paper [14] concentrates in particular on the existence of a giant component in the random graphs $\mathcal{G}(n, M)$ and $\mathcal{G}(n, p)$, that is, a connected component that contains a constant proportion of all $n$ vertices in the graph. In the $\mathcal{G}(n, M)$ model, $M$ edges are chosen among all $\left(\begin{array}{l}n \\ 2\end{array}\right)$ pairs of vertices uniformly at random to form a random graph with exactly $M$ edges, while in the $\mathcal{G}(n, p)$ model every pair of vertices forms an edge with probability $p$ in the final graph independently from all other pairs (or equivalently, $G \in \mathcal{G}(n, p)$ is a random subgraph of the complete graph on $n$ vertices after $p$-percolation of its edges). In [14, Erdoos and Rényi proved the following (by now very classical) result: for any $\varepsilon>0$, if $M \leq(1-\varepsilon) n / 2$, then all connected components in the random graph $G \in \mathcal{G}(n, M)$ have $O(\log n)$ vertices asymptotically almost surely (a.a.s.), while if $M \geq(1+\varepsilon) n / 2$, then the largest component in the random graph $G \in \mathcal{G}(n, M)$ contains $\Omega(n)$ vertices but the second largest contains only $O(\log n)$ vertices a.a.s. Later Bollobás $[5]$ and Euczak [20] made a precise analysis of the more complicated regime when $M=n / 2+o(n)$ and exhibited a critical window around $M=n / 2$ of width of order $\Theta\left(n^{2 / 3}\right)$, in which a number of connected components with $\Theta\left(n^{2 / 3}\right)$ vertices in each happen to coexist a.a.s. All results above have natural analogues for $\mathcal{G}(n, p)$. Aldous [2] later made a beautiful connection between the sizes of the connected components in the critical regime in $\mathcal{G}(n, p)$ and the zeros of a Brownian motion with a suitable drift.

In fact, percolation of finite graphs was considered in many particular cases. Another classical example is the hypercube in dimension $n$, denoted by $H_{n}$. The graph $H_{n}$ has vertices $\{0,1\}^{n}$ and two vertices $u$ and $v$ are connected by an edge if they differ in exactly one entry. In [16] Erdôs and Spencer showed that if $p \leq(1-\varepsilon) / n$, then a.a.s. $p$-percolation of $H_{n}$ leaves a graph with largest component, containing at most $o\left(2^{n}\right)$ vertices, and they conjectured that a component with $\Omega\left(2^{n}\right)$ vertices is a.a.s. present if $p \geq(1+\varepsilon) / n$. This conjecture was confirmed by Ajtai, Komlós and Szemerédi in [1]. A following series of papers of Bollobás, Kohayakawa and Łuczak [6], Borgs, Chayes, van der Hofstad, Slade and Spencer [7, 8, 9], van der Hofstad and Nachmias [23], and Hulshof and Nachmias [17] provides a deep understanding of the critical percolation on the hypercube in high dimension. 
To the best of our knowledge there were two attempts for generalising the sharp threshold phenomenon for the existence of a giant component for large families of finite graphs. Chung, Horn and Lu [10] showed the existence of a sharp threshold under several conditions involving the spectrum of the adjacency matrix of the base graph. Sadly, their conditions are not satisfied for the hypercube $H_{n}$, see [12]. Alon, Benjamini and Stacey [3] proved the existence of a sharp threshold in expanders of uniformly bounded degree. Here as well, although the hypercube $H_{n}$ has indeed good expansion properties [22], its degree goes to infinity with $n$.

Our goal in this paper is to generalise the existence of a sharp threshold for the appearance of a giant component for Cartesian products of graphs under two assumptions: on the maximum degrees and on the isoperimetric constants of the graphs in the product. In particular, our result ensures the existence of a sharp threshold for the appearance of a giant component for the Cartesian product of any sequence of $n$ connected graphs with uniformly bounded orders, the hypercube $H_{n}$ being a particular case of the latter. We believe it is worth making the connection with Joos [19], who studies the threshold probability for connectivity of percolated sparse graphs and, as a corollary, completely solves the problem for Cartesian powers of a graph $G$.

\subsection{Notation and terminology}

For every positive integer $n$, we denote by $[n]$ the set $\{1,2, \ldots, n\}$. In this paper, for any three positive real numbers $a, b, c$, by $a / b c$ or $a / b \cdot c$ we mean $a /(b c)$.

For a graph $G$, the order of $G$ is the cardinality of its vertex set $V(G)$, and the size of $G$ is the cardinality of its edge set $E(G)$. For a vertex $v \in V(G)$, we denote by $\operatorname{deg}_{G}(v)$, or just $\operatorname{deg}(v)$, the degree of $v$ in $G$, and by $C C_{G}(v)$, or just $C C(v)$, the connected component of $v$ in $G$. Then, the average degree of $G$ is defined by

$$
\bar{d}(G)=\frac{1}{|V(G)|} \sum_{v \in V(G)} \operatorname{deg}_{G}(v) .
$$

The maximum degree of a graph $G$ is denoted $\Delta(G)$, and the order of the largest connected component in $G$ is denoted $L_{1}(G)$. Finally, for any graph $G$ with $|V(G)| \geq 2$, the isoperimetric constant of $G$ is given by

$$
i(G)=\min _{\substack{S \subseteq V(G) ; \\ 1 \leq|S| \leq|V(G)| / 2}} \frac{|\partial S|}{|S|},
$$

where $\partial S=\partial_{G} S$ is the set of edges in $G$ between a vertex in $S$ and a vertex in $V(G) \backslash S$. Clearly if $L_{1}(G)<|V(G)|$, then $i(G)=0$. For a set $S \subseteq V(G)$, we also denote by $N_{G}(S)$, or just $N(S)$, the set of vertices in $G$ at graph distance 1 from $S$ in $G$, and also $N_{G}[S]$, or simply $N[S]$, is defined as $S \cup N(S)$.

For any sequence of $n$ graphs $G_{1}, G_{2}, \ldots, G_{n}$, the Cartesian product of $G_{1}, G_{2}, \ldots, G_{n}$, denoted by $G_{1} \square G_{2} \square \ldots \square G_{n}$ or $\square_{i \in[n]} G_{i}$, is the graph with vertex set

$$
\left\{\left(v_{1}, v_{2}, \ldots, v_{n}\right) \mid \forall i \in[n], v_{i} \in V\left(G_{i}\right)\right\}
$$

and edge set

$$
\left\{\left(u_{1}, u_{2}, \ldots, u_{n}\right)\left(v_{1}, v_{2}, \ldots, v_{n}\right) \mid \exists i \in[n], \forall j \neq i, u_{j}=v_{j} \text { and } u_{i} v_{i} \in E\left(G_{i}\right)\right\} .
$$

A $p$-percolation of a graph $G$ is a random process in which every edge in $G$ is retained with probability $p$ and deleted with probability $1-p$, independently from all other edges. If an edge is retained, we say that it is open, and if it is deleted, we say that it is closed. The graph consisting of all open edges is a random subgraph of $G$, which we denote by $G_{p}$.

For a sequence of probability spaces $\left(\Omega_{n}, \mathcal{F}_{n}, \mathbb{P}_{n}\right)_{n \geq 1}$ and a sequence of events $\left(A_{n}\right)_{n \geq 1}$, where $A_{n} \in \mathcal{F}_{n}$ for every $n \geq 1$, we say that $\left(A_{n}\right)_{n \geq 1}$ happens asymptotically almost surely or a.a.s. if $\lim _{n \rightarrow+\infty} \mathbb{P}_{n}\left(A_{n}\right)=1$. The sequence of events $\left(A_{n}\right)_{n \geq 1}$ itself is said to be asymptotically almost sure or again a.a.s. 
Our main result, Theorem 1.1, is of asymptotic nature. Therefore, below we use the well-known asymptotic notations $o, O, \Omega$ and $\Theta$. For two functions $f, g: \mathbb{N} \rightarrow \mathbb{R}^{+}$we also write $f(n) \ll g(n)$ or $g(n) \gg f(n)$ if $f(n)=o(g(n))$. Moreover, if the limit variable is not $n$, we will indicate this using lower indices such as $O_{x}$.

\subsection{Our result}

Throughout the paper we fix two absolute constants $\gamma>0$ and $C \in \mathbb{N}$ (that is, these constants do not depend on any other parameters in the sequel). Let $\left(G_{n, j}\right)_{n \in \mathbb{N}, j \in[n]}$ be finite connected graphs with at least one edge such that, for every $n \in \mathbb{N}$ and $j \in[n]$ :

1. $\Delta\left(G_{n, j}\right) \leq C$, and

2. $i\left(G_{n, j}\right) \geq n^{-\gamma}$.

Define $G_{[n]}=\square_{j \in[n]} G_{n, j}$. In the sequel we write $G_{j}$ for $G_{n, j}, G$ for $G_{[n]}$, and $\bar{d}$ for $\bar{d}\left(G_{[n]}\right)$, hopefully taking enough care to ensure that no confusion arises due to this abuse of notation. We insist that we reserve the notation $G_{p}$ for the subgraph of $G$ after $p$-percolation, so $G_{p}$ is not part of $\left(G_{j}\right)_{j \in[n]}$. For any vertex $v \in V(G)$ we denote by $C C_{p}(v)=C C_{G_{p}}(v)$. Since a vertex $v=\left(v_{1}, \ldots, v_{n}\right) \in V(G)$ has degree $\sum_{j=1}^{n} \operatorname{deg}_{G_{j}}\left(v_{j}\right)$, we conclude that

$$
\bar{d}=\sum_{j=1}^{n} \bar{d}\left(G_{j}\right) .
$$

Now we present the main result of the paper.

Theorem 1.1. Fix $\varepsilon \in(0,1)$. In the above setup:

a) if $p=(1-\varepsilon) / \bar{d}$, then a.a.s. $L_{1}\left(G_{p}\right) \leq \exp \left(-\frac{\varepsilon^{2} n}{9 C^{2}}\right)|V(G)|$, and

b) if $p=(1+\varepsilon) / \bar{d}$, then there is a positive constant $c_{1}=c_{1}(\varepsilon, \gamma, C)$ such that a.a.s. $L_{1}\left(G_{p}\right) \geq c_{1}|V(G)|$.

Remark 1.2. One may replace the constant $C$ with a function $n^{\alpha}$ in condition 1, where $\alpha=\alpha(\gamma)$ is a positive constant, and Theorem 1.1 will still be valid. We present the proof only of the given more simplified version of Theorem 1.1 for two reasons: first, the idea of the proof is the same and this more general version would only make the exposition more technical, and second, we believe that even this more general framework does not fully explain the existence of a sharp threshold for the giant component problem for product graphs.

Let us give a quick overview of the proof of Theorem 1.1. The first point concerns the study of two subcritical exploration processes. The first one ensures an upper bound on the order of the union of all components, containing at least one vertex of "high" degree. The second process deals with the remaining "low" degree vertices conditionally on the edges, exposed during the first process, and is therefore directly dominated by a subcritical branching process. The proof of the second point is inspired by the special case of the hypercube, studied in [1. It relies on consecutively constructing connected components with larger and larger polynomial orders via the technique of two-round exposure (or rather multi-round exposure in our case). Once the correct polynomial order is attained, we show by the same technique that the condition 2 on the isoperimetric constants of the graphs in the product ensures that a constant proportion of the above components are merged together in $G_{p}$ a.a.s.

The paper is organised as follows. In Section 2 we introduce several preliminary results. Then, in Section 3 we prove Theorem $1.1 \mathrm{a}$ ), and in Section 4 we prove Theorem $1.1 \mathrm{~b}$ ). Finally, Section 5 is dedicated to a discussion and a couple of open questions. 


\section{Preliminaries}

\subsection{Probabilistic preliminaries}

Chernoff's inequality: We first state a version of the famous Chernoff's inequality, see e.g. ([18], Theorem 2.1).

Lemma 2.1 ([18], Theorem 2.1). Let $X \sim \operatorname{Bin}(n, p)$ be a Binomial random variable with parameters $n$ and $p$. Then, for any $t \geq 0$ we have

$$
\begin{aligned}
& \mathbb{P}(X \geq \mathbb{E}[X]+t) \leq \exp \left(-\frac{t^{2}}{2(\mathbb{E}[X]+t / 3)}\right), \text { and } \\
& \mathbb{P}(X \leq \mathbb{E}[X]-t) \leq \exp \left(-\frac{t^{2}}{2 \mathbb{E}[X]}\right) .
\end{aligned}
$$

The bounded difference inequality: The following well-known inequality is a simple consequence of the Azuma-Hoeffding martingale inequality, see [18] or also [21] for an improvement.

Theorem 2.2 (The bounded difference inequality, see e.g. [18]). Consider a sequence $\left(X_{i}\right)_{i \in[n]} \in \prod_{i \in[n]} \Lambda_{i}$ of $n$ independent random variables. Fix a function $f: \prod_{i \in[n]} \Lambda_{i} \rightarrow \mathbb{R}$ and suppose that there exist $\left(C_{i}\right)_{i \in[n]}$ such that, for every $i \in[n],\left(x_{j}\right)_{j \in[n]} \in \prod_{j \in[n]} \Lambda_{j}$ and $x_{i}^{\prime} \in \Lambda_{i}$, we have

$$
\left|f\left(x_{1}, \ldots, x_{i-1}, x_{i}, x_{i+1}, \ldots, x_{n}\right)-f\left(x_{1}, \ldots, x_{i-1}, x_{i}^{\prime}, x_{i+1}, \ldots, x_{n}\right)\right| \leq C_{i} .
$$

Then, for every $t \geq 0$,

$$
\mathbb{P}\left(\left|f\left(X_{1}, \ldots, X_{n}\right)-\mathbb{E}\left[f\left(X_{1}, \ldots, X_{n}\right)\right]\right| \geq t\right) \leq 2 \exp \left(-\frac{t^{2}}{2 \sum_{i \in[n]} C_{i}^{2}}\right) .
$$

The Bienaymé-Galton-Watson random tree: By now a very well-known and studied model is the Bienaymé-Galton-Watson random tree, or $B G W$ tree. Let $\nu$ be a probability distribution over $\mathbb{N} \cup\{0\}$ and let $X$ be a random variable with distribution $\nu$. The BGW tree with progeny distribution $\nu$ is constructed as follows. Starting from a vertex $v_{0}$ (the root), every vertex gives birth (just once) to a random number of children, distributed according to $\nu$ and independent from all other vertices in the tree. The BGW tree is subcritical if $\mathbb{E}[X]<1$, supercritical if $\mathbb{E}[X]>1$, and critical otherwise. It is a basic fact in the theory of branching processes that a subcritical BGW tree is almost surely finite while a supercritical BGW tree has strictly positive probability to be infinite. The next lemma makes the first statement more precise by giving a probabilistic estimate on the size of a subcritical BGW tree, see e.g. (44, Theorem 2.3.2).

Lemma 2.3 ([4], Theorem 2.3.2). Let $T$ be a subcritical $B G W$ tree such that $\mathbb{E}\left[s^{X}\right]<+\infty$ for some $s>1$. Define

$$
h_{\nu}=\sup _{\theta>0}(\theta-\log (\mathbb{E}[\exp (\theta X)])) .
$$

Then, for every $k \geq 1$ we have

$$
\mathbb{P}(|V(T)| \geq k) \leq \exp \left(-k h_{\nu}\right)
$$

Fix $\varepsilon \in(0,1)$. We will use the above result in the particular case when $X \sim \operatorname{Bin}(n, p)$ with $p=(1-\varepsilon) / n$. We have

$$
\mathbb{E}[\exp (\theta X)]=\sum_{i=0}^{n}\left(\begin{array}{l}
n \\
k
\end{array}\right) \exp (\theta k) p^{k}(1-p)^{n-k}=(1-p+\exp (\theta) p)^{n} .
$$


Then, using that $\log (1+\theta)=\theta+O_{\theta}\left(\theta^{2}\right)$, as $n \rightarrow+\infty$ we have

$$
\begin{aligned}
h_{X} & =\sup _{\theta>0}(\theta-n \log (1-p+\exp (\theta) p)) \\
& =\sup _{\theta>0}\left(\theta-n\left(-p+\exp (\theta) p+O\left(1 / n^{2}\right)\right)\right) \\
& =\sup _{\theta>0}(\theta+1-\varepsilon-(1-\varepsilon) \exp (\theta)+O(1 / n)) .
\end{aligned}
$$

Since $\exp (\theta)=1+\theta+O_{\theta}\left(\theta^{2}\right)$, the latter quantity tends to a constant $\phi=\phi(\varepsilon)>0$ as $n \rightarrow+\infty$, where

$$
\phi(\varepsilon)=\sup _{\theta>0}(\theta+1-\varepsilon-(1-\varepsilon) \exp (\theta)) .
$$

Corollary 2.4. The BGW tree $T$ with progeny distribution $\operatorname{Bin}(n,(1-\varepsilon) / n)$ satisfies

$$
\mathbb{P}(|V(T)| \geq k) \leq \exp (-(1+o(1)) k \phi) .
$$

In particular, for every $\varepsilon \in(0,1)$ there is $k_{0}=k_{0}(\varepsilon) \in \mathbb{N}$ such that for every $k \geq k_{0}$ we have

$$
\mathbb{P}(|V(T)| \geq k) \leq \exp (-k \phi / 2)
$$

\subsection{Combinatorial preliminaries}

The isoperimetric constant of a product graph: Recall that $G$ is a graph, defined as a Cartesian product of the graphs $G_{1}, G_{2}, \ldots, G_{n}$. The next result, due to Chung and Tetali [11], makes a connection between the isoperimetric constant of $G$ and the isoperimetric constants of $\left(G_{k}\right)_{k \in[n]}$, see also Tillich [22] for a slight improvement.

Theorem 2.5 ([11], Theorem 2).

$$
\frac{1}{2} \min _{k \in[n]} i\left(G_{k}\right) \leq i(G) \leq \min _{k \in[n]} i\left(G_{k}\right)
$$

We directly deduce the following corollary.

Corollary 2.6. Under condition 圆 on $\left(G_{k}\right)_{k \in[n]}$ we have $n^{-\gamma} / 2 \leq i(G)$.

The largest connected component and "balanced" empty cuts: The following easy observation makes a connection between empty cuts in a graph and the size of the largest connected component.

Observation 2.7. Fix $k \in \mathbb{N}$. Let $H$ be a graph with $h$ vertices and let $\mathcal{C}_{1}, \ldots, \mathcal{C}_{k}$ be disjoint connected subgraphs of $H$ such that $\cup_{j \in[k]} V\left(\mathcal{C}_{j}\right)=V(H)$. Suppose that for any set $J \subseteq[k]$ such that $\left|\cup_{j \in J} V\left(\mathcal{C}_{j}\right)\right| \in$ $[h / 3,2 h / 3]$, there exists an edge in $H$ between a vertex in $\cup_{j \in J} \mathcal{C}_{j}$ and a vertex in $\cup_{j \in[k] \backslash J} \mathcal{C}_{j}$. Then, there is a connected component of $H$ that contains more than $h / 3$ vertices.

Proof. We argue by contradiction. Suppose that $H$ contains $m$ connected components and each of them has order at most $h / 3$. Then, consider the graphs $H_{0}=\varnothing, H_{1}, H_{2}, \ldots, H_{m}$, where for every $i \in[m]$ we define $H_{i}$ to be the union of $H_{i-1}$ and some connected component in the graph $H \backslash H_{i-1}$. Since $\left|V\left(H_{m}\right)\right|=h$ and for every $i \in[m],\left|V\left(H_{i}\right)\right|-\left|V\left(H_{i-1}\right)\right| \leq h / 3$, by discrete continuity there is $\ell \in[m]$ such that $\left|V\left(H_{\ell}\right)\right| \in[h / 3,2 h / 3]$. This is a contradiction with the assumption in the statement for the family $\left\{\mathcal{C}_{j}: \mathcal{C}_{j} \cap H_{\ell}=\emptyset\right\}$. The observation is proved. 


\section{Proof of Theorem $1.1 \mathrm{a})$ - the subcritical regime}

We begin with a proof of Theorem 1.1 a). Our first step will be to estimate the number of vertices of $G$ of degree at least $(1+\varepsilon / 2) \bar{d}$. For every $i \in[n]$, let $X_{i}$ be the degree of a uniformly chosen vertex in $G_{i}$. For every $i \in[n]$, define $S_{i}=X_{1}+X_{2}+\cdots+X_{i}$. Since $\mathbb{E}\left[S_{n}\right]=\bar{d}$ by (11) and for every $i \in[n]$ we have $\Delta\left(G_{i}\right) \leq C$, we conclude by the bounded difference inequality (Theorem 2.2) that

$$
\mathbb{P}\left(\left|S_{n}-\bar{d}\right| \geq \varepsilon \bar{d} / 2\right) \leq 2 \exp \left(-\frac{\varepsilon^{2} \bar{d}^{2} / 4}{2 C^{2} n}\right) \leq 2 \exp \left(-\frac{\varepsilon^{2} n}{8 C^{2}}\right)
$$

The second inequality comes from the fact that $\bar{d} \geq n$ : indeed, by (11) we have $\bar{d}=\sum_{i=1}^{n} \bar{d}\left(G_{i}\right)$, and every graph among $\left(G_{i}\right)_{i \in[n]}$ is connected and therefore its average degree is at least 1 . Therefore, the number

of vertices in $G$ with degree more that $(1+\varepsilon / 2) \bar{d}$ is at most a $2 \exp \left(-\frac{\varepsilon^{2} n}{8 C^{2}}\right)$-proportion of all vertices of $G$.

Proof of Theorem $1.1 \mathrm{a}$ ). First, we prove that the number of vertices connected via a path in $G_{p}$ to a vertex of degree at least $(1+\varepsilon / 2) \bar{d}$ in $G$ is at most $\exp \left(-\frac{\varepsilon^{2} n}{9 C^{2}}\right)|V(G)|$ a.a.s. Indeed, let $U$ be the set of vertices of degree at least $(1+\varepsilon / 2) \bar{d}$ in $G$. Then, by (3) we have $|U| \leq \exp \left(-\frac{\varepsilon^{2} n}{8 C^{2}}\right)|V(G)|$. We consider the following stochastic process. Let $U_{0}=N_{G_{p}}(U)$, and for every positive integer $k$ we inductively define $U_{k}=N_{G_{p}}\left(U_{k-1}\right) \backslash\left(U \cup U_{0} \cup \cdots \cup U_{k-1}\right)$. Since for every set $V \subseteq V(G) \backslash U$ we have $|\partial V| \leq(1+\varepsilon / 2) \bar{d}|V|$, we have that, for every $k \geq 1$,

$$
\mathbb{E}\left[\left|U_{k}\right| \mid U_{k-1}\right] \leq p\left|\partial U_{k-1}\right| \leq p(1+\varepsilon / 2) \bar{d}\left|U_{k-1}\right| \leq(1-\varepsilon / 2)\left|U_{k-1}\right| .
$$

We conclude that for every $k \geq 1$,

$$
\mathbb{E}\left[\left|U_{k}\right|\right] \leq(1-\varepsilon / 2)^{k} \mathbb{E}\left[\left|U_{0}\right|\right] \leq(1-\varepsilon / 2)^{k} C n|U| .
$$

Thus, we get by Markov's inequality that

$$
\mathbb{P}\left(\exists k \geq 1,\left|U_{k}\right| \geq(1-\varepsilon / 2)^{k / 2} C n^{2}|U|\right) \leq \sum_{k \geq 1} \mathbb{P}\left(\left|U_{k}\right| \geq(1-\varepsilon / 2)^{k / 2} C n^{2}|U|\right) \leq \sum_{k \geq 1} \frac{(1-\varepsilon / 2)^{k / 2}}{n}=o(1) .
$$

We deduce that the union of all connected components of $G_{p}$, containing at least one vertex of $U$, contains at most $\sum_{k \geq 1}(1-\varepsilon)^{k / 2} C n^{2}|U|=\Theta\left(n^{2}|U|\right)=o(|V(G)|)$ vertices a.a.s.

Denote the set of vertices in all explored connected components by $\bar{U}$. Here, an edge of $G$ is explored if the fact that it is open or not has been revealed, and a connected component is explored if all edges it contains have been explored and are open, while all edges on its boundary have been explored and are closed. After exploring all connected components of $G_{p}$, containing at least one vertex in $U$, we are left with unexplored edges, incident only to vertices of degree less than $(1+\varepsilon / 2) \bar{d}$ in $G$. We prove that in the remainder of $G_{p}$ there is a.a.s. no connected component of order more than $\lceil 4 \log |V(G)| / \phi(\varepsilon / 2)\rceil$, with $\phi$ defined in (2). Indeed, choose any vertex $v$ and start an exploration process of its connected component $C C_{p}(v)$ in $G_{p}$. Note that any vertex in $V(G) \backslash \bar{U}$ is incident to less than $(1+\varepsilon / 2) \bar{d}$ unexplored edges. Thus, the number of edges in $C C_{p}(v)$ is stochastically dominated by the number of explored edges in a BGW tree $T$ with progeny distribution $\operatorname{Bin}(\lfloor(1+\varepsilon / 2) \bar{d}\rfloor, p)$. Since $\bar{d} \rightarrow+\infty$ with $n$ and

$$
p=\frac{1-\varepsilon}{\bar{d}} \leq \frac{1-\varepsilon / 2}{(1+\varepsilon / 2) \bar{d}} \leq \frac{1-\varepsilon / 2}{\lfloor(1+\varepsilon / 2) \bar{d}\rfloor},
$$


by Corollary 2.4 we get that for every $k \geq 1$ and for every $n$ large enough

$$
\mathbb{P}\left(\left|V\left(C C_{p}(v)\right)\right| \geq k\right) \leq \mathbb{P}(|V(T)| \geq k) \leq \exp \left(-\frac{k \phi(\varepsilon / 2)}{2}\right) .
$$

Choosing $k=k_{0}:=\lceil 4 \log |V(G)| / \phi(\varepsilon / 2)\rceil$, we get that with probability at most $1 /|V(G)|^{2}, C C_{p}(v)$ contains at least $k_{0}$ vertices. A union bound over all vertices in $V(G) \backslash \bar{U}$ implies that with probability at most $1 /|V(G)|$, the largest component in $G_{p}$, containing no vertex in $U$, is of order at least $k_{0}$. Since

$$
\exp \left(-\frac{\varepsilon^{2} n}{9 C^{2}}\right)|V(G)| \geq 2^{-n / 2}|V(G)| \geq \sqrt{|V(G)|} \gg \log |V(G)|
$$

the proof is finished.

\section{Proof of Theorem $1.1 \mathrm{~b}$ ) - the supercritical regime}

The remainder of the paper will be directed towards proving Theorem 1.1 b). The main technique, wellknown under the name two-round exposure or sprinkling, has by now become a classical tool in the field of random graphs. It states that the graph $G_{p}$ may be realised as a union of two random graphs on the same vertex set $G_{p_{1}}$ and $G_{p_{2}}$, sampled independently from each other, where $\left(1-p_{1}\right)\left(1-p_{2}\right)=1-p$. Indeed, the probability that an edge in $G$ does not appear in $G_{p}$ is $1-p$, while by independence the probability that an edge in $G$ does not appear in $G_{p_{1}} \cup G_{p_{2}}$ is $\left(1-p_{1}\right)\left(1-p_{2}\right)$. Moreover, in both $G_{p}$ and $G_{p_{1}} \cup G_{p_{2}}$, different edges appear independently from each other.

In our case, inspired by [1, we show that one may choose $p_{1}$ and $p_{2}$ appropriately so that a.a.s. $G_{p_{1}}$ consists of a number of connected components of order at least $\Omega\left(n^{k}\right)$ for some large enough positive integer $k$, which contain a constant proportion of all vertices of $G$. Then, at the second stage, we show that a.a.s. a constant proportion of all such component merge in a connected component of size $\Theta(|V(G)|)$.

In the sequel, $\operatorname{deg}(v)$ will refer to the degree of a vertex $v$ in $G$.

Observation 4.1. For some $i \in[n]$, let

$$
v_{1}=\left(w_{1}, \ldots, w_{i-1}, u_{1}, w_{i+1}, \ldots, w_{n}\right) \text { and } v_{2}=\left(w_{1}, \ldots, w_{i-1}, u_{2}, w_{i+1}, \ldots, w_{n}\right)
$$

be two vertices in $G$. Then, $\left|\operatorname{deg}\left(v_{1}\right)-\operatorname{deg}\left(v_{2}\right)\right| \leq C-1$.

Proof. We have $\operatorname{deg}\left(v_{1}\right)-\operatorname{deg}\left(v_{2}\right)=\operatorname{deg}_{G_{i}}\left(u_{1}\right)-\operatorname{deg}_{G_{i}}\left(u_{2}\right)$. The claim follows since the graph $G_{i}$ is connected and has maximum degree at most $C$.

Corollary 4.2. If $C \geq 2$, two vertices $v_{1}$ and $v_{2}$ in $G$ are at graph distance at least $\frac{\left|\operatorname{deg}\left(v_{1}\right)-\operatorname{deg}\left(v_{2}\right)\right|}{C-1}$.

Let $D$ be the set of vertices of degree at most $(1-\varepsilon / 2) \bar{d}$ in $G$.

Lemma 4.3. Fix $\varepsilon \in(0,0.1)$ and $p \geq(1+7 \varepsilon / 8) / \bar{d}$. There is a constant $c_{1}=c_{1}(\varepsilon)>0$ such that, for every large enough $n$, every vertex in $G$ of degree at least $(1-\varepsilon / 4) \bar{d}$ participates in a connected component of $G_{p}$ of size at least $\varepsilon \bar{d} / 4 C$ with probability at least $c_{1}$.

Proof. Fix a vertex $v_{0}$ satisfying $\operatorname{deg}_{G}\left(v_{0}\right) \geq(1-\varepsilon / 4) \bar{d}$ and start an exploration process of the connected component of $v_{0}$ in $G_{p}$ as follows. We divide the vertices of $G_{p}$ in several categories: active, when the edges, incident to this vertex, have not been explored from the vertex itself, but it has been attained via a path from $v_{0}$, passive, if the vertex was active before but the edges in its neighbourhood have been explored from it, and processed, when the vertex is either active or passive. For example, in the beginning only the vertex $v_{0}$ is active and there are no passive vertices. A reformulation of the statement of the lemma is that, by starting an exploration process of $G_{p}$ from $v_{0}$, with probability at least $c_{1}$ at least $\varepsilon \bar{d} / 4 C$ vertices 
will be processed in the end. Start by exploring all edges in $G$, going out of $v_{0}$, and make all neighbours of $v_{0}$ in $G_{p}$ active. Then, make $v_{0}$ passive and find an active vertex $v_{1}$, if it exists. Then, explore all edges incident to $v_{1}$ in $G$ and make all neighbours of $v_{1}$ in $G_{p}$ that have not yet been processed active. Then, make $v_{1}$ passive and find an active vertex $v_{2}$, if it exists, etc. Continue with the exploration until either all or at least $\varepsilon \bar{d} / 4 C$ vertices in the connected component of $v_{0}$ in $G_{p}$ have been processed.

Fix an integer $n \geq 8 C^{2} / \varepsilon$. If $C \geq 2$, by Corollary 4.2 every vertex of degree at least $(1-\varepsilon / 4) \bar{d}$ in $G$ is at distance at least $\varepsilon \bar{d} / 4(C-1) \geq \varepsilon \bar{d} / 4 C+1$ from $D$. The same holds if $C=1$ since $D=\emptyset$ then. Fix any integer $k \in[2, \varepsilon \bar{d} / 4 C]$ (this interval is non-empty since $\bar{d} \geq n \geq 8 C / \varepsilon$ ). Under the assumption that at most $k$ vertices in $G_{p}$ have been made passive before exploring the neighbourhood of a particular active vertex $u$, at least

$$
\operatorname{deg}_{G}(u)-1-C-(C-1)(k-1) \geq \operatorname{deg}_{G}(u)-C k \geq(1-\varepsilon / 2) \bar{d}-\varepsilon \bar{d} / 4 \geq(1-3 \varepsilon / 4) \bar{d}
$$

neighbours of $u$ have never been processed before (here, $1+C+(C-1)(k-1) \leq C k$ is an upper bound of the total number of processed vertices after $k$ steps, and $\operatorname{deg}_{G}(u) \geq(1-\varepsilon / 2) \bar{d}$ since $u$ is at distance at most $k \leq \varepsilon \bar{d} / 4 C$ from $v_{0}$ that satisfies $\left.\operatorname{deg}_{G}\left(v_{0}\right) \geq(1-\varepsilon / 4) \bar{d}\right)$. Therefore, until the number of processed vertices is at most $\varepsilon \bar{d} / 4 C$, the number of edges of $G$, incident to the currently explored vertex $u$ and leading to vertices which have never been processed before, is at least $(1-3 \varepsilon / 4) \bar{d}$. We may conclude that the exploration of the connected component of $v_{0}$ in $G_{p}$, up to the moment of finding $\lceil\varepsilon \bar{d} / 4 C\rceil$ processed vertices, stochastically dominates the exploration of a BGW tree with progeny distribution $\operatorname{Bin}\left(\lceil(1-3 \varepsilon / 4) \bar{d}\rceil, \frac{1+7 \varepsilon / 8}{\bar{d}}\right)$. For every $\varepsilon \leq 0.1$, the BGW tree with these parameters is supercritical since

$$
\lceil(1-3 \varepsilon / 4) \bar{d}\rceil \cdot \frac{1+7 \varepsilon / 8}{\bar{d}} \geq 1+\frac{\varepsilon}{8}-\frac{21 \varepsilon^{2}}{32}>1,
$$

and therefore it has probability $c_{1}=c_{1}(\varepsilon)>0$ to grow to infinity. Thus, with probability at least $c_{1}$, the exploration of $G_{p}$ from $v_{0}$ leads to at least $\varepsilon \bar{d} / 4 C$ processed vertices, which proves the lemma.

Following [1, we call a connected subgraph of $G_{p}$ a cell. Note that a connected component of $G_{p}$ is a cell, but a cell does not have to be a connected component of $G_{p}$ itself. Fix the constant $c_{1}=c_{1}(\varepsilon)$ given by Lemma 4.3. We say that a vertex $v$ is a neighbour of a set of vertices $A$ in a graph $H$ if there is a vertex $u \in A$, which is a neighbour of $v$ in $H$. Let $P_{p}$ be the following property of a vertex $v$ of $G$ :

the vertex $v$ is a neighbour in $G$ to at least $c_{1} \varepsilon n / 64 C$ disjoint cells in $G_{p}$, each of order at least $\varepsilon n / 8 C$.

Lemma 4.4. Fix $\varepsilon \in(0,0.1)$ and $p=(1+\varepsilon) / \bar{d}$. There is a constant $c_{2}=c_{2}(\varepsilon)>0$ such that, for every large enough $n$, every vertex in $G$ of degree at least $(1-\varepsilon / 8) \bar{d}$ has property $P_{p}$ with probability at least $1-\exp \left(-c_{2} n\right)$.

Proof. Fix a vertex $v$ satisfying $\operatorname{deg}_{G}(v) \geq(1-\varepsilon / 8) \bar{d}$. Let $v=\left(v_{1}, v_{2}, \ldots, v_{n}\right)$. For every $i \in[n]$, let $u_{i}$ be a neighbour of $v_{i}$ in $G_{i}$, and define

$$
H_{i}=\left(\underset{j \in[i-1]}{\square} v_{j}\right) \square u_{i} \square\left(\underset{k \in[n] \backslash[i]}{\square} G_{k}\right) .
$$

Thus, the graph $H_{i}$ is a conveniently chosen projection of $G$, which is isomorphic to $\underset{k \in[n] \backslash[i]}{\square} G_{k}$. Also, for every $i \in[n]$, let $\hat{v}_{i}=\left(v_{1}, \ldots, v_{i-1}, u_{i}, v_{i+1}, \ldots, v_{n}\right)$. Then, we claim that, for every $i \leq i_{\max }:=$ 
$\lfloor\varepsilon n / 16 C\rfloor-1$, the vertex $\hat{v}_{i}$ has degree at least $(1-\varepsilon / 8) \bar{d}-C(i+1) \geq(1-\varepsilon / 4) \bar{d}\left(H_{i}\right)$ in $H_{i}$. Indeed,

$$
\begin{aligned}
\left(1-\frac{\varepsilon}{8}\right) \bar{d}-C(i+1) & \geq\left(1-\frac{\varepsilon}{8}\right) \bar{d}\left(H_{i}\right)-C(i+1) \\
& \geq\left(1-\frac{\varepsilon}{8}\right) \bar{d}\left(H_{i}\right)-\frac{\varepsilon n}{16} \\
& \geq\left(1-\frac{\varepsilon}{4}\right) \bar{d}\left(H_{i}\right)+\frac{\varepsilon}{8}\left(\bar{d}\left(H_{i}\right)-\frac{n}{2}\right) \\
& \geq\left(1-\frac{\varepsilon}{4}\right) \bar{d}\left(H_{i}\right)+\frac{\varepsilon}{8}\left(n-i-\frac{n}{2}\right) \geq\left(1-\frac{\varepsilon}{4}\right) \bar{d}\left(H_{i}\right) .
\end{aligned}
$$

Moreover, by the choice of $i$ we also have

$$
(1+\varepsilon) \bar{d}\left(H_{i}\right) \geq(1+\varepsilon)(\bar{d}-C(i+1)) \geq(1+\varepsilon) \bar{d}-2 C(i+1) \geq\left(1+\frac{7 \varepsilon}{8}\right) \bar{d} .
$$

Thus, $p=(1+\varepsilon) / \bar{d} \geq(1+7 \varepsilon / 8) / \bar{d}\left(H_{i}\right)$, and we may apply Lemma 4.3 to the vertex $\hat{v}_{i}$ in $H_{i}$ and deduce that the probability that $\hat{v}_{i}$ participates in a cell in $H_{i}$ of order at least $\varepsilon \bar{d}\left(H_{i}\right) / 4 C \geq \varepsilon n / 8 C$ is at least $c_{1}$. Since the graphs $\left(H_{i}\right)_{i \in\left[i_{\max }\right]}$ are disjoint, the events

$$
\left(A_{i}:=\left\{\text { the connected component of } \hat{v}_{i} \text { in } H_{i} \text { contains at least } \varepsilon n / 8 C \text { vertices }\right\}\right)_{i \in\left[i_{\max }\right]}
$$

are independent and each of them happens with probability at least $c_{1}$. Thus, by Chernoff's inequality (Lemma 2.1) for $\left(\mathbb{1}_{A_{i}}\right)_{i \in i_{\max }}$ with $t=\mathbb{E}\left[\sum_{i=1}^{i_{\max }} \mathbb{1}_{A_{i}}\right] / 2 \geq c_{1} i_{\max } / 2$, the vertex $v$ is incident to at least $c_{1} i_{\max } / 2 \geq c_{1}(\varepsilon n / 32 C) / 2=c_{1} \varepsilon n / 64 C$ disjoint cells in its neighbourhood in $G$ with probability at least $1-\exp (-t / 8) \geq 1-\exp \left(-c_{1} \varepsilon n / 512 C\right)$. Thus, $c_{2}=c_{1} \varepsilon / 512 C$ satisfies our requirements, and the lemma is proved.

Fix the constant $c_{2}=c_{2}(\varepsilon)>0$, given by Lemma 4.4 .

Corollary 4.5. Fix $\varepsilon \in(0,0.1)$ and $p=(1+\varepsilon) / \bar{d}$. Then, every vertex $v \in V(G)$ with $\operatorname{deg}_{G}(v) \geq(1-\varepsilon / 16) \bar{d}$ satisfies the following property with probability at least $1-\exp \left(-\left(c_{2}+o(1)\right) n\right): v$ is a neighbour in $G$ to at least $c_{1} \varepsilon n / 64 C$ disjoint cells of $G_{p}$, each containing at least $\varepsilon n / 17 C$ vertices with the property $P_{p}$.

Proof. Fix any vertex $v$ satisfying $\operatorname{deg}_{G}(v) \geq(1-\varepsilon / 16) \bar{d}$. By Lemma 4.4 it has probability at least $1-\exp \left(-c_{2} n\right)$ to have property $P_{p}$. We condition on this event. Then, for every cell $\mathcal{C}$ among the first $\left\lceil c_{1} \varepsilon n / 64 C\right\rceil$ disjoint neighbouring cells of size at least $\varepsilon n / 8 C$, corresponding to $v$, put a label $\ell_{v}$ on the $\lceil\varepsilon n / 17 C\rceil$ vertices of $\mathcal{C}$ that are closest to $v$ in the graph $G_{p}$ (if some set of vertices is at the same distance to $v$ in $G_{p}$, make an arbitrary choice which of them to label, if necessary). Thus, for every vertex $u$ which has received a label $\ell_{v}$ we have $d_{G}(u, v) \leq d_{G_{p}}(u, v) \leq\lceil\varepsilon n / 17 C\rceil$. Moreover, by Corollary 4.2 for every large enough $n$ we have $|\operatorname{deg}(u)-\operatorname{deg}(v)| \leq C\lceil\varepsilon n / 17 C\rceil \leq \varepsilon n / 16$ and so $\operatorname{deg}(u) \geq \operatorname{deg}(v)-\varepsilon n / 16 \geq \bar{d}-\varepsilon \bar{d} / 16-\varepsilon n / 16 \geq(1-\varepsilon / 8) \bar{d}$.

Note that a total of at most $\left\lceil c_{1} \varepsilon n / 64 C\right\rceil \cdot(\varepsilon n / 16 C)=\Theta\left(n^{2}\right)$ vertices will receive the label $\ell_{v}$, and furthermore by Lemma 4.4 each of these vertices has property $P_{p}$ with probability at least $1-\exp \left(-c_{2} n\right)$. Then, conditionally on the event that $v$ has property $P_{p}$, any vertex $u$ with label $\ell_{v}$ has property $P_{p}$ with probability

$$
\mathbb{P}\left(u \text { has } P_{p} \mid v \text { has } P_{p}\right)=\frac{\mathbb{P}\left(u \text { and } v \text { have } P_{p}\right)}{\mathbb{P}\left(v \text { has } P_{p}\right)} \geq 1-2 \exp \left(-c_{2} n\right)
$$

Thus, the vertex $v$ satisfies the property from the statement of the corollary with probability at least

$$
1-\sum_{u \text { has label } \ell_{v}} \mathbb{P}\left(u \text { does not have } P_{p} \mid v \text { has } P_{p}\right) \geq 1-\Theta\left(n^{2}\right) \exp \left(-c_{2} n\right)=1-\exp \left(-\left(c_{2}+o(1)\right) n\right) .
$$

The corollary is proved. 
With the help of Corollary 4.5, we are ready to improve on Lemma 4.4 by showing that every vertex of sufficiently high degree in $G$ has, with high probability, many neighbours in $G$, which participate in connected components of $G_{p}$ of order $\Omega\left(n^{2}\right)$. Denote $c_{1}^{\prime}=\min \left(c_{1}(\varepsilon / 2), 1\right)$ and $c_{2}^{\prime}=c_{2}(\varepsilon / 2)$.

Lemma 4.6. Fix $\varepsilon \in(0,0.1)$ and $p=(1+\varepsilon) / \bar{d}$. There are constants $c_{3}=c_{3}(\varepsilon)>0$ and $c_{4}=c_{4}(\varepsilon)>0$ such that for every vertex $v$ satisfying $\operatorname{deg}_{G}(v) \geq(1-\varepsilon / 32) \bar{d}$, the following property holds with probability at least $1-\exp \left(-\left(c_{4}+o(1)\right) n\right): v$ is adjacent (in $G$ ) to at least $c_{3} n$ vertices, participating in connected components of $G_{p}$ of order at least $c_{3} \varepsilon n^{2} / 32 C$.

Proof. We use the technique of two-round exposure with $p_{1}=(1+\varepsilon / 2) / \bar{d}$ and $p_{2}$ given by the equation $\left(1-p_{1}\right)\left(1-p_{2}\right)=(1-p)$. Since $\bar{d} \rightarrow+\infty$ with $n, p_{2}=(\varepsilon / 2+o(1)) / \bar{d}$, so for every large enough $n$ we have $p_{2} \geq \varepsilon / 4 \bar{d}$.

Fix a vertex $v$ satisfying $\operatorname{deg}_{G}(v) \geq(1-\varepsilon / 32) \bar{d}$. By Corollary 4.5, applied with $\varepsilon / 2$ instead of $\varepsilon$, we get that with probability $1-\exp \left(-\left(c_{2}^{\prime}+o(1)\right) n\right)$ the vertex $v$ is a neighbour (in $G$ ) to at least $c_{1}^{\prime} \varepsilon n / 128 C$ disjoint cells of $G_{p_{1}}$, each containing at least $\varepsilon n / 34 C$ vertices with the property $P_{p_{1}}$. We condition on this event. Fix any such vertex $v$ and let $\mathcal{C}_{1}, \ldots, \mathcal{C}_{k}$ be the cells, which correspond to $v$ in the above statement, with $k \geq c_{1}^{\prime} \varepsilon n / 128 C$.

Fix an arbitrary cell, say $\mathcal{C}_{1}$, and let $u_{1}, u_{2}, \ldots, u_{m}$ be vertices in $\mathcal{C}_{1}$ which satisfy the property $P_{p_{1}}$, where $m=\lfloor\varepsilon n / 34 C\rfloor$. Moreover, assume that for every vertex $u_{i}$ and every cell $\mathcal{C}$ in a fixed set of $\left\lceil c_{1}^{\prime} \varepsilon n / 128 C\right\rceil$ disjoint cells, which witnesses that $u_{i}$ satisfies the property $P_{p_{1}}, \mathcal{C}$ contains exactly $\lceil\varepsilon n / 16 C\rceil$ vertices (clearly any connected graph $H$ contains a connected subgraph of any order between 1 and $|V(H)|$ ). For every $i \in[m]$, we associate the above set of cells to the vertex $u_{i}$.

Now, we consider the independent percolation of the edges in $G$ with parameter $p_{2}$. This is our second round. We do the following exploration process. List $u_{1}, \ldots, u_{m}$ in this order and start exploring their neighbourhoods one by one. If $u_{1}$ connects (during the second round percolation with parameter $p_{2}$ ) to a neighbouring cell of size $\lceil\varepsilon n / 16 C\rceil$, which was associated to it, then name this cell $\mathcal{C}_{1}^{\prime}$. Then, go to $u_{2}$. If $\mathcal{C}_{1}^{\prime}$ was well defined, there are at most two cells $\mathcal{C}^{\prime}$ among the ones, associated to $u_{2}$, such that $\left|\mathcal{C}^{\prime} \cap \mathcal{C}_{1}^{\prime}\right| \geq\lceil\varepsilon n / 16 C\rceil / 2$. Let $\left(w_{j}^{2}\right)_{j \in[2]}$ be the two vertices, which connect $u_{2}$ to neighbouring cells, associated to $u_{2}$ and with the largest intersection with $\mathcal{C}_{1}^{\prime}$. Then, if $u_{2}$ connects to a neighbouring cell associated to it via an edge, different from $u_{2} w_{1}^{2}$ and $u_{2} w_{2}^{2}$, name this cell $\mathcal{C}_{2}^{\prime}$ and go to $u_{3}$. Then, since $\left|\mathcal{C}_{1}^{\prime} \cup \mathcal{C}_{2}^{\prime}\right| \leq 2\lceil\varepsilon n / 16 C\rceil$, there are at most 4 cells $\mathcal{C}^{\prime}$, associated to $u_{3}$, for which $\left|\mathcal{C}^{\prime} \cap\left(\mathcal{C}_{1}^{\prime} \cup \mathcal{C}_{2}^{\prime}\right)\right| \geq\lceil\varepsilon n / 16 C\rceil / 2$. Let $\left(w_{j}^{3}\right)_{j \in[4]}$ be the four vertices, which connect $u_{3}$ to neighbouring cells, associated to $u_{3}$ and with the largest possible intersection with $\mathcal{C}_{1}^{\prime} \cup \mathcal{C}_{2}^{\prime}$. Then, if $u_{3}$ connects to a neighbouring cell associated to it via an edge, different from $\left(u_{3} w_{j}^{3}\right)_{j \in[4]}$, name this cell $\mathcal{C}_{3}^{\prime}$ and continue with $u_{4}$, ets.

Suppose that in the moment of exploring the neighbourhood of the vertex $u_{i}$ we came across the cells $\mathcal{C}_{i_{1}}^{\prime}, \mathcal{C}_{i_{2}}^{\prime}, \ldots, \mathcal{C}_{i_{j}}^{\prime}$ for some $1 \leq i_{1}<\cdots<i_{j} \leq i-1$. Then,

$$
\left|\bigcup_{1 \leq s \leq j} \mathcal{C}_{i_{s}}^{\prime}\right|=\sum_{1 \leq s \leq j}\left|\mathcal{C}_{i_{s}}^{\prime} \backslash\left(\mathcal{C}_{i_{1}}^{\prime} \cup \cdots \cup \mathcal{C}_{i_{s-1}}^{\prime}\right)\right| \geq \frac{j \varepsilon n}{32 C}
$$

Also, for every $i \leq i_{0}:=\left\lfloor c_{1}^{\prime} \varepsilon n / 512 C\right\rfloor$ (by definition of $c_{1}^{\prime}$ we have $i_{0} \leq m$ ), there are at least $c_{1}^{\prime} \varepsilon n / 128 C-$ $2 c_{1}^{\prime} \varepsilon n / 512 C \geq c_{1}^{\prime} \varepsilon n / 256 C$ cells, associated to the vertex $u_{i}$, which do not intersect the union of cells $\mathcal{C}_{i_{1}}^{\prime}, \mathcal{C}_{i_{2}}^{\prime}, \ldots, \mathcal{C}_{i_{j}}^{\prime}$ in more than $\lceil\varepsilon n / 16 C\rceil / 2$ vertices. Thus, for every large enough $n$ and every $i \leq i_{0}, u_{i}$ has probability at least $p_{3}:=p_{2} c_{1}^{\prime} \varepsilon n / 256 C \geq c_{1}^{\prime} \varepsilon^{2} / 1024 C^{2}$ to connect to a cell, which does not intersect the union of $\mathcal{C}_{i_{1}}^{\prime}, \mathcal{C}_{i_{2}}^{\prime}, \ldots, \mathcal{C}_{i_{j}}^{\prime}$ in more than $\lceil\varepsilon n / 16 C\rceil / 2$ vertices. We conclude that the indicator functions of the events

$$
\left(\left\{u_{i} \text { connects to a cell } \mathcal{C}^{\prime} \text { associated to it and such that }\left|\mathcal{C}^{\prime} \cap\left(\cup_{1 \leq \ell \leq j} \mathcal{C}_{i_{\ell}}\right)\right|<\frac{\lceil\varepsilon n / 16 C\rceil}{2}\right\}\right)_{i \in\left[i_{0}\right]}
$$

stochastically dominate a family of i.i.d. random variables $\left(B_{i}\right)_{i \in\left[i_{0}\right]}$ with Bernoulli distribution with parameter $p_{3}$. By a direct application of Chernoff's inequality (Lemma 2.1) we conclude that for every 
large enough $n$ and $c_{3}=c_{3}(\varepsilon)=\left(c_{1}^{\prime}\right)^{2} \varepsilon^{3} / 2^{21} C^{3}$

$$
\mathbb{P}\left(\sum_{i \in\left[i_{0}\right]} B_{i} \leq \frac{p_{3} i_{0}}{2}\right) \leq \exp \left(-\frac{p_{3} i_{0}}{8}\right) \leq \exp \left(-\frac{\left(c_{1}^{\prime} \varepsilon n / 2\right) \cdot\left(c_{1}^{\prime} \varepsilon^{2}\right)}{8 \cdot 512 C \cdot 1024 C^{2}}\right)=\exp \left(-c_{3} n / 4\right) .
$$

Thus, for every large enough $n$ and every $\ell \in[k]$, the connected component of $\mathcal{C}_{\ell}$ in $G_{p}=G_{p_{1}} \cup G_{p_{2}}$ contains at least $\left(p_{3} i_{0} / 2\right) \cdot(\varepsilon n / 32 C) \geq c_{3} \varepsilon n^{2} / 32 C$ vertices with probability at least $1-\exp \left(-c_{3} n / 4\right)$. A union bound over all $k \leq C n$ cells shows that, for every large enough $n$ and for every vertex $v$ satisfying $\operatorname{deg}_{G}(v) \geq(1-\varepsilon / 32) \bar{d}$ and with at least $c_{1}^{\prime} \varepsilon n / 128 C$ neighbouring cells of order $\lceil\varepsilon n / 16 C\rceil$ in $G_{p_{1}}$, with probability at least $1-C n \exp \left(-c_{3} n / 4\right)$ each of the connected components of $\mathcal{C}_{1}, \mathcal{C}_{2}, \ldots, \mathcal{C}_{k}$ in $G_{p}$ is of order at least $c_{3} \varepsilon n^{2} / 32 C$.

Now, let $c_{4}=\min \left(c_{2}^{\prime}, c_{3} / 4\right)$. Then, with probability at least $1-\exp \left(-\left(c_{4}+o(1)\right) n\right)$, the vertex $v$ is incident to at least $c_{3} n$ vertices, which participate in connected components of $G_{p}$ of size at least $c_{3} \varepsilon n^{2} / 32 C$. The lemma is proved.

Up to this moment, we ensured the existence of a large number of connected components of order at least $\Theta\left(n^{2}\right)$ in $G_{p}$. Recall that $\gamma$ is a positive constant such that, for every $j \in[n], n^{-\gamma} \leq i\left(G_{j}\right)$. If $\gamma \in(0,1)$, we are ready to complete the proof of Theorem 1.1. However, for larger values we will need to show more. In the sequel we ensure that there are a lot of components of size $\Omega\left(n^{\gamma^{\prime}+2}\right)$ in $G_{p}$ for some $\gamma^{\prime}>\gamma$. The aim of the next lemma is to iterate the procedure of Lemma 4.4. Corollary 4.5] and Lemma 4.6 to provide the a.a.s. existence of these larger connected components. Unlike the results we presented above, we will mostly rely on the asymptotic notations $\Theta$ and $\Omega$ in the proof of Lemma 4.7 rather than give explicit constants to simplify the presentation, having in mind that very similar but more precise formulations of the claims below were already presented in detail.

Lemma 4.7. Fix any integer $k \geq 2$, any $\varepsilon \in(0,0.1)$ and $p=(1+\varepsilon) / \bar{d}$. Then, there are positive constants $\beta_{k} \geq 32, C_{k}=C_{k}(\varepsilon), C_{k}^{\prime}=C_{k}^{\prime}(\varepsilon), C_{k}^{\prime \prime}=C_{k}^{\prime \prime}(\varepsilon)$ such that for every vertex $v$ satisfying $\operatorname{deg}_{G}(v) \geq\left(1-\varepsilon / \beta_{k}\right) \bar{d}$, the following property holds with probability at least $1-\exp \left(-\left(C_{k}^{\prime \prime}+o(1)\right) n\right): v$ is adjacent (in $\left.G\right)$ to at least $C_{k}^{\prime} n$ vertices, participating in connected components in $G_{p}$ of order at least $C_{k} n^{k}$.

Proof. We argue by induction. By Lemma 4.6 the statement is true for $k=2$ with parameters $\beta_{2}=$ $32, C_{2}=c_{3} \varepsilon / 32 C, C_{2}^{\prime}=c_{3}$ and $C_{2}^{\prime \prime}=c_{4}$ for every $\varepsilon \in(0,0.1)$.

Suppose that the statement is satisfied for some $k-1 \geq 2$. Fix $p_{0}=(1+\varepsilon / 4) / \bar{d}, p_{0}^{\prime}=(\varepsilon / 4+o(1)) / \bar{d}$ and $p_{1}=(1+\varepsilon / 2) / \bar{d}$ so that $\left(1-p_{0}\right)\left(1-p_{0}^{\prime}\right)=1-p_{1}$. Moreover, for any vertex $v$ in $G$, denote by $P_{p, k}$ the following property:

the vertex $v$ is a neighbour in $G$ of $\Omega(n)$ disjoint cells in $G_{p}$, each of order $\Omega\left(n^{k}\right)$.

The next claim is an analogue of Lemma 4.4, so we give only the main points of the proof.

Claim 4.8. Every vertex of degree at least $\left(1-\varepsilon / 8 \beta_{k-1}\right) \bar{d}$ in $G$ has property $P_{p_{1}, k-1}$ with probability $1-\exp (-\Omega(n))$.

Proof. We follow the proof of Lemma 4.4. Fix a vertex $v=\left(v_{1}, v_{2}, \ldots, v_{n}\right)$ satisfying $\operatorname{deg}_{G}(v) \geq(1-$ $\left.\varepsilon / 8 \beta_{k-1}\right) \bar{d}$. For every $i \in[n]$, let $u_{i}$ be a neighbour of $v_{i}$ in $G_{i}$. Denote $\hat{v}_{i}=\left(v_{1}, \ldots, v_{i-1}, u_{i}, v_{i+1}, \ldots, v_{n}\right)$ and

$$
H_{i}:=\left(\underset{j \in[i-1]}{\square} v_{j}\right) \square u_{i} \square\left(\underset{k \in[n] \backslash[i]}{\square} G_{k}\right) .
$$

Then, for every $\varepsilon \in(0,0.1)$ there exists a positive constant $\bar{c}_{k-1}=\bar{c}_{k-1}(\varepsilon) \leq 1 / 2$ such that, for every $i \leq \bar{c}_{k-1} n$, the degree of $\hat{v}_{i}$ in $H_{i}$ is at least $\left(1-\varepsilon / 4 \beta_{k-1}\right) \bar{d}\left(H_{i}\right)=\left(1-(\varepsilon / 4) / \beta_{k-1}\right) \bar{d}\left(H_{i}\right)$. By the induction hypothesis, applied with $\varepsilon / 4, p_{0}, \hat{v}_{i}$ and $H_{i}$ (which is isomorphic to the product of at least $n-i \geq n / 2$ of the graphs $\left.\left(G_{j}\right)_{j \in[n]}\right)$, the vertex $\hat{v}_{i}$ is incident to at least $\Theta(n / 2)$ vertices, participating 
in connected components in $H_{i, p_{0}}$ of order $\Omega\left((n / 2)^{k-1}\right)$ with probability $1-\exp (-\Omega(n / 2))$. (Note that although $\Omega(n)=\Omega(n / 2)$ and $\Omega\left(n^{k-1}\right)=\Omega\left((n / 2)^{k-1}\right)$, we add the constants to indicate that the graph $H_{i}$ is a product of less that $n$, but at least $n / 2$ graphs. When considered appropriate, similar implicit indications are given below as well).

It remains to notice that the graphs $\left(H_{i}\right)_{1 \leq i \leq \bar{c}_{k-1} n}$ are disjoint and therefore the vertices $\left(\hat{v}_{i}\right)_{1 \leq i \leq \bar{c}_{k-1} n}$ connect to cells of order $\Omega\left((n / 2)^{k-1}\right)$ in $\left(H_{i}\right)_{1 \leq i \leq \bar{c}_{k-1} n}$ respectively at the second round percolation with parameter $p_{0}^{\prime}$ independently and with probability $p_{0}^{\prime} \Omega(n / 2)=\Omega(1)$. Thus, by Chernoff's inequality (Lemma 2.1) we deduce that the vertex $v$ has $\Omega(1) \cdot \bar{c}_{k-1} n / 2=\Omega(n)$ neighbours, which participate into disjoint cells of $G_{p_{1}}=G_{p_{0}} \cup G_{p_{0}^{\prime}}$ of size $\Omega\left((n / 2)^{k-1}\right)$ with probability $1-\exp \left(-\Omega(1) \cdot \bar{c}_{k-1} n / 8\right)=$ $1-\exp (-\Omega(n))$. The proof is completed.

Claim 4.9. Every vertex $v$ with $\operatorname{deg}_{G}(v) \geq\left(1-\varepsilon / 16 \beta_{k-1}\right) \bar{d}$ satisfies the following property with probability $1-\exp (-\Omega(n))$ : the vertex $v$ is a neighbour in $G$ to $\Omega(n)$ disjoint cells of $G_{p_{1}}$, each containing $\Omega(n)$ vertices with the property $P_{p_{1}, k-1}$.

Proof. We follow the proof of Corollary 4.5. Fix any vertex $v$ satisfying $\operatorname{deg}_{G}(v) \geq\left(1-\varepsilon / 16 \beta_{k-1}\right) \bar{d}$. Since $\beta_{k-1} \geq 32$, by Lemma $4.4 v$ has probability $1-\exp (-\Omega(n))$ to have property $P_{p_{1}}$. We condition on this event. Then, for every cell $\mathcal{C}$ among the $\Omega(n)$ disjoint neighbouring cells of order at least $\lceil\varepsilon n / 16 C\rceil$, corresponding to $v$, put a label $\ell_{v}$ on the $\left\lceil\varepsilon n /\left(16 C \beta_{k-1}+1\right)\right\rceil$ vertices of $\mathcal{C}$ that are closest to $v$ in the graph $G_{p_{1}}$ (if some set of vertices is at the same distance to $v$ in $G_{p}$, make an arbitrary choice which of them to label, if necessary). Thus, for every vertex $u$ which has received a label $\ell_{v}$ we have $d_{G}(u, v) \leq d_{G_{p_{1}}}(u, v) \leq\left\lceil\varepsilon n /\left(16 C \beta_{k-1}+1\right)\right\rceil$. Moreover, by Corollary 4.2 for every large enough $n$ we have

$$
|\operatorname{deg}(u)-\operatorname{deg}(v)| \leq C\left\lceil\varepsilon n /\left(16 C \beta_{k-1}+1\right)\right\rceil \leq \varepsilon n / 16 \beta_{k-1}
$$

and so

$$
\operatorname{deg}(u) \geq \operatorname{deg}(v)-\varepsilon n / 16 \beta_{k-1} \geq \bar{d}-\varepsilon \bar{d} / 16 \beta_{k-1}-\varepsilon n / 16 \beta_{k-1} \geq\left(1-\varepsilon / 8 \beta_{k-1}\right) \bar{d} .
$$

Note that a total of $O\left(n^{2}\right)$ vertices will receive the label $\ell_{v}$, and furthermore by Claim 4.8 each of these vertices has property $P_{p_{1}, k-1}$ with probability $1-\exp (-\Omega(n))$. Then, conditionally on the event that $v$ has property $P_{p_{1}}$, any vertex $u$ with label $\ell_{v}$ has property $P_{p_{1}, k-1}$ with probability

$$
\mathbb{P}\left(u \text { has } P_{p_{1}, k-1} \mid v \text { has } P_{p_{1}}\right)=\frac{\mathbb{P}\left(u \text { has } P_{p_{1}, k-1} \text { and } v \text { has } P_{p_{1}}\right)}{\mathbb{P}\left(v \text { has } P_{p_{1}}\right)} \geq 1-2 \exp (-\Omega(n))=1-\exp (-\Omega(n)) .
$$

Thus, the vertex $v$ satisfies the property from the statement of the claim with probability at least

$$
1-\sum_{u \text { has label } \ell_{v}} \mathbb{P}\left(u \text { does not have } P_{p_{1}, k-1} \mid v \text { has } P_{p_{1}}\right) \geq 1-O\left(n^{2}\right) \exp (-\Omega(n))=1-\exp (-\Omega(n)) .
$$

The claim is proved.

The finish the proof of the lemma, we follow the ideas of the proof of Lemma 4.6. We use once again the technique of two-round exposure with $p_{1}=(1+\varepsilon / 2) / \bar{d}$ and $p_{2}=(\varepsilon / 2+o(1)) / \bar{d}$ such that $\left(1-p_{1}\right)\left(1-p_{2}\right)=(1-p)$.

Fix a vertex $v$ satisfying $\operatorname{deg}_{G}(v) \geq\left(1-\varepsilon / 32 \beta_{k-1}\right) \bar{d}$. By Claim 4.9 we get that, for some positive constant $\hat{C}_{k-1}=\hat{C}_{k-1}(\varepsilon)$, with probability $1-\exp (-\Omega(n))$ the vertex $v$ is a neighbour (in $G$ ) to at least $\hat{C}_{k-1} n$ disjoint cells of $G_{p_{1}}$, each containing $\Omega(n)$ vertices with the property $P_{p_{1}, k-1}$. Let us condition on this event, and let $\mathcal{C}_{1}, \ldots, \mathcal{C}_{t}$ be the cells, which correspond to $v$ in the above statement, where $t$ is an integer satisfying $\hat{C}_{k-1} n \leq t \leq C n$.

Fix an arbitrary cell among $\mathcal{C}_{1}, \ldots, \mathcal{C}_{t}$, say $\mathcal{C}_{1}$, and let $u_{1}, u_{2}, \ldots, u_{m}$ be vertices in $\mathcal{C}_{1}$ which satisfy the property $P_{p_{1}, k-1}$, where $m=\Omega(n)$. Moreover, assume that for every vertex $u_{i}$ and every cell $\mathcal{C}$ among a fixed set of $\Omega(n)$ disjoint cells, which witnesses that $u_{i}$ satisfies the property $P_{p_{1}, k-1}, \mathcal{C}$ contains exactly 
$\left\lceil\hat{C}_{k-1}^{\prime} n^{k-1}\right\rceil$ vertices, where $\hat{C}_{k-1}^{\prime}$ is a positive constant depending only on $k$ and $\varepsilon$. By the very same exploration procedure as in the proof of Lemma 4.6 we show that with probability $1-\exp (-\Omega(n))$ the connected component of the cell $\mathcal{C}_{1}$ in $G_{p}=G_{p_{1}} \cup G_{p_{2}}$ contains at least $s=\Omega(n)$ cells $\mathcal{C}_{1}^{\prime}, \mathcal{C}_{2}^{\prime}, \ldots, \mathcal{C}_{s}^{\prime}$ such that, for every $i \in[s]$,

$$
\left|V\left(\mathcal{C}_{i}^{\prime} \backslash \cup_{j \in[i-1]} \mathcal{C}_{j}^{\prime}\right)\right| \geq \frac{\left\lceil\hat{C}_{k-1}^{\prime} n^{k-1}\right\rceil}{2} .
$$

Recall that $\mathcal{C}_{1}^{\prime}, \mathcal{C}_{2}^{\prime}, \ldots, \mathcal{C}_{s}^{\prime}$ are cells, associated to different vertices among $u_{1}, u_{2}, \ldots, u_{m}$, and therefore these are not necessarily disjoint. Thus, bounding from below the number of vertices in every new cell that does not participate in the union of the previous ones is crucial to attain the lower bound on the size of the union.

Since the above reasoning applies to each of the cells $\mathcal{C}_{1}, \mathcal{C}_{2}, \ldots, \mathcal{C}_{t}$, associated to $v$, and $t=\Theta(n)$, we conclude by union bound that $v$ satisfies property $P_{p, k}$ with probability $1-\Theta(n) \exp (-\Omega(n))=1-$ $\exp (-\Omega(n))$, which finishes the proof (the constants $C_{k}, C_{k}^{\prime}$ and $C_{k}^{\prime \prime}$ are hidden in the $\Omega$ notation but $\beta_{k}$ could be defined recursively by $\beta_{k}=32 \beta_{k-1}$, so in particular $\left.\beta_{k}=32^{k-1}\right)$.

We are ready to prove Theorem 1.1 b). Fix $k=\lceil 1+\gamma\rceil+3$. Up to now, we have ensured the existence of a number of cells in $G_{p}$, which contain at least $C_{k} n^{k}$ vertices. In the sequel, let $\hat{C}_{k}=C_{k}(\varepsilon / 2), \hat{C}_{k}^{\prime}=C_{k}^{\prime}(\varepsilon / 2)$ and $\hat{C}_{k}^{\prime \prime}=C_{k}^{\prime \prime}(\varepsilon / 2)$.

Proof of Theorem $1.1 \mathrm{~b})$. It is sufficient to prove the claim for every $\varepsilon \in(0,0.1)$. Once again, we consider two-round exposure of $G_{p}=G_{p_{1}} \cup G_{p_{2}}$ with $p_{1}=(1+\varepsilon / 2) / \bar{d}$ and $p_{2}=(\varepsilon / 2+o(1)) / \bar{d}$. By Lemma 4.7 and Markov's inequality with probability at least $1-\exp \left(-\left(\hat{C}_{k}^{\prime \prime} / 2+o(1)\right) n\right)$ all but at most an $\exp \left(-\hat{C}_{k}^{\prime \prime} n / 2\right)-$ proportion of all vertices of degree at least $\left(1-(\varepsilon / 2) / \beta_{k}\right) \bar{d}$ in $G$ have at least $\hat{C}_{k}^{\prime} n$ neighbours, which participate in connected components of $G_{p_{1}}$ of order at least $\hat{C}_{k} n^{k}$. We condition on this event. Then, the number of edges, adjacent to vertices in connected components of $G_{p_{1}}$ of order at least $\hat{C}_{k} n^{k}$ is at least $(1+o(1)) \hat{C}_{k}^{\prime} n|V(G)| / 2$ (recall that asymptotically almost all vertices of $G$ have degree at least $\left.\left(1-(\varepsilon / 2) / \beta_{k}\right) \bar{d}\right)$. Since every vertex has degree at most $C n$ in $G$, there are at least $(1+o(1)) \hat{C}_{k}^{\prime}|V(G)| / 2 C$ vertices of $G$ in connected components of $G_{p_{1}}$ of order at least $\hat{C}_{k} n^{k}$.

We prove that the following property holds with probability $1-\exp \left(-\Omega\left(|V(G)| / n^{k}\right)\right)$ :

the vertices in all connected components in $G_{p_{1}}$ of order at least $\hat{C}_{k} n^{k}$ cannot be partitioned into two sets, $V_{1}$ and $V_{2}$, such that ||$V_{1}|-| V_{2}|| \leq\left(\left|V_{1}\right|+\left|V_{2}\right|\right) / 3$ (or equivalently $\left|V_{1}\right| / 2 \leq\left|V_{2}\right| \leq\left|V_{1}\right|$ up to symmetry considerations) so that there is no path in $G_{p}$ between $V_{1}$ and $V_{2}$.

On the above event, by Observation 2.7 we may directly conclude that the largest connected component of $G_{p}$ contains at least $\left(\left|V_{1}\right|+\left|V_{2}\right|\right) / 3 \geq(1+o(1)) \hat{C}_{k}|V(G)| / 6 C$ vertices, which would finish the proof of Theorem $1.1 \mathrm{~b}$ ).

Since the number of connected components of $G_{p_{1}}$ of order at least $\hat{C}_{k} n^{k}$ is $O\left(|V(G)| / n^{k}\right)$, there are $2^{O\left(|V(G)| / n^{k}\right)}$ ways to partition these components into two sets. We will be interested only in partitions $\left(V_{1}, V_{2}\right)$ such that $\left|V_{1}\right| / 2 \leq\left|V_{2}\right| \leq\left|V_{1}\right|$. Consider two cases:

1. $N_{G}\left[V_{1}\right] \cap N_{G}\left[V_{2}\right] \geq|V(G)| / n^{k-2}$, and

2. $N_{G}\left[V_{1}\right] \cap N_{G}\left[V_{2}\right]<|V(G)| / n^{k-2}$.

In the first case, we know by our conditioning that $(1+o(1))|V(G)| / n^{k-2}$ of the vertices in $N_{G}\left[V_{1}\right] \cap$ $N_{G}\left[V_{2}\right]$ have at least $\hat{C}_{k}^{\prime} n / 2$ neighbours (in $G$ ) in either $V_{1}$ or $V_{2}$, or in both. Therefore, the probability that a fixed vertex in $N_{G}\left[V_{1}\right] \cap N_{G}\left[V_{2}\right]$ connects $V_{1}$ and $V_{2}$ at the second round percolation with parameter $p_{2}$ is at least $\left(1-\left(1-p_{2}\right)^{\hat{C}_{k}^{\prime} n / 2}\right) \cdot p_{2}=\Theta(1 / n)$. Moreover, the above events are independent for different 
vertices in $N_{G}\left[V_{1}\right] \cap N_{G}\left[V_{2}\right]$. Therefore, the probability that $V_{1}$ and $V_{2}$ do not get connected at the second round percolation with parameter $p_{2}$ is at most

$$
(1-\Theta(1 / n))^{(1+o(1))|V(G)| / n^{k-2}}=\exp \left(-\Theta\left(|V(G)| / n^{k-1}\right)\right) \ll 2^{-O\left(|V(G)| / n^{k}\right)} .
$$

In the second case, since the number of edges between $V_{2}$ and $N_{G}\left(V_{2}\right)$ is at least $i(G)\left|V_{2}\right|$ by assumption, by Corollary 2.6 there are at least $i(G)\left|V_{2}\right| / C n \geq n^{-1-\gamma}\left|V_{2}\right| / 2 C$ vertices in $V(G) \backslash V_{2}$, adjacent to $V_{2}$ in G. But

$$
\left|N_{G}\left[V_{1}\right] \cap N_{G}\left[V_{2}\right]\right|<|V(G)| / n^{k-2} \ll n^{-1-\gamma}\left|V_{2}\right| / 2 C,
$$

so by our conditioning each of the $(1+o(1)) n^{-\gamma-1}\left|V_{2}\right| / 2 C$ vertices in $N_{G}\left[V_{2}\right] \backslash N_{G}\left[V_{1}\right]$ have at least $\hat{C}_{k}^{\prime} n$ edges towards $V_{2}$ in $G$. On the other hand, since $\left|V_{1}\right| \geq\left|V_{2}\right|$ and $\left|V_{1} \cap N_{G}\left[V_{2}\right]\right|=o\left(\left|V_{1}\right|\right)$, we have by Corollary 2.6 that there are at least

$$
i(G) \min \left(\left|N_{G}\left[V_{2}\right] \backslash N_{G}\left[V_{1}\right]\right|,(1+o(1))\left|V_{1}\right|\right)=\Omega\left(n^{-\gamma}|V(G)|\right)
$$

edges, going out of $N_{G}\left[V_{2}\right] \backslash N_{G}\left[V_{1}\right]$. One may directly deduce that there are $\Omega\left(n^{-\gamma}|V(G)|\right) / C n=$ $\Omega\left(n^{-\gamma-1}|V(G)|\right)$ disjoint edges, which have one endvertex in $N_{G}\left[V_{2}\right] \backslash N_{G}\left[V_{1}\right]$ and one endvertex in $V(G) \backslash N_{G}\left[V_{2}\right]$. Since all but $\exp (-\Omega(n))|V(G)|$ vertices have at least $\hat{C}_{k}^{\prime} n$ edges towards $V_{1} \cup V_{2}$ by our conditioning, we deduce that there are $\Omega\left(n^{-\gamma-1}|V(G)|\right)$ disjoint edges $u v$ in $G$ such that $u$ has at least $\hat{C}_{k}^{\prime} n$ edges towards $V_{1}$ and $v$ has at least $\hat{C}_{k}^{\prime} n$ edges towards $V_{2}$. We conclude that for any such edge $u$ and $v$ there is a path from $V_{1}$ through $u$ and $v$ towards $V_{2}$ with probability $\left(1-\left(1-p_{2}\right)^{\hat{C}_{k}^{\prime} n}\right) \cdot p_{2} \cdot\left(1-\left(1-p_{2}\right)^{\hat{C}_{k}^{\prime} n}\right)=\Theta(1 / n)$. Therefore, the probability that $V_{1}$ and $V_{2}$ do not get connected at the second round percolation with parameter $p_{2}$ is at most

$$
(1-\Theta(1 / n))^{\Omega\left(|V(G)| / n^{\gamma+1}\right)}=\exp \left(-\Omega\left(|V(G)| / n^{\gamma+2}\right)\right) \ll 2^{-O\left(|V(G)| / n^{k}\right)} .
$$

We conclude the proof of Theorem $1.1 \mathrm{~b}$ ) by a union bound over all $2^{O\left(|V(G)| / n^{k}\right)}$ partitions of the components of size at least $\hat{C}_{k} n^{k}$ in $G_{p_{1}}$.

\section{$5 \quad$ Discussion and further questions}

In this paper we proved that there is a sharp threshold for the existence of a giant component after percolation of the product graph $G=G_{1} \square \ldots \square G_{n}$ under the assumptions that $\max _{j \in[n]} \Delta\left(G_{j}\right)$ is uniformly bounded from above by a constant and $\min _{j \in[n]} i\left(G_{j}\right)$ decays to zero at most polynomially fast. As Remark 1.2 points out, at the price of a more technical exposition Theorem 1.1 may be generalised for graphs with slowly increasing degrees. Except for simplicity, we spared the details also because we believe that Theorem 1.1 may also be proved in an even more general setting.

To begin with, we were not able to find convincing counterexamples of the sharp threshold phenomenon without the maximum degrees assumption. In the proof of Theorem 1.1 presented above, this assumption was used in most of our lemmas.

Question 5.1. Can one prove an analogue of Theorem 1.1 without the assumption on the maximum degrees of $\left(G_{j}\right)_{j \in[n]}$ ?

Concerning the assumption on the decay of the isoperimetric constants, we show that it cannot be removed entirely. Consider the graph $G$ where $G_{1}=G_{2}=\cdots=G_{n-1}$, each containing two vertices $(0$ and 1$)$ a single edge $(01)$, and $G_{n}$ being a cycle of length $2^{2^{n}}$. Then, all vertices in $G$ will have degree $n+1$. Fix $p=2 /(n+1)$. Note that for any edge $u v$ of $G_{n}$ we have that the probability that each of the edges $((x, u)(x, v))_{x \in\{0,1\}^{n-1}}$ of $G$ disappears after $p$-percolation is $(1-2 /(n+1))^{2^{n-1}}=$ $\exp \left(-(1+o(1)) 2^{n} /(n+1)\right)$. Thus, on average many of the sets of edges $((x, u)(x, v))_{x \in\{0,1\}^{n-1}} \subseteq E(G)$ for different edges $u v$ of $G_{n}$ disappear a.a.s. after $p$-percolation, so no giant component exists since for any two edges $u_{1} v_{1}$ and $u_{2} v_{2}$ of $G_{n}$, the edge set $\left\{\left(x, u_{1}\right)\left(x, v_{1}\right)\right\}_{x \in\{0,1\}^{n-1}} \cup\left\{\left(x, u_{2}\right)\left(x, v_{2}\right)\right\}_{x \in\{0,1\}^{n-1}} \subseteq E(G)$ forms a cut in $G$. Although somewhat trivial, this example leads to another logical question. 
Question 5.2. Can one prove an analogue of Theorem 1.1 if $\min _{j \in[n]} i\left(G_{j}\right)$ decreases faster than a polynomial function of $n$ ?

Of course, graph products other than the Cartesian product exist as well. It might be interesting to study the appearance of a giant component with respect to them.

Question 5.3. Can one prove analogous results for other graph products?

\section{Acknowledgements}

The author would like to thank Dieter Mitsche, Guillem Perarnau and Ivailo Hartarsky for several useful remarks, and Felix Joos for turning my attention to the reference [19]. I am also grateful to the two anonymous referees for a number of important comments and suggestions.

\section{References}

[1] M. Ajtai, J. Komlós, and E. Szemerédi. Largest random component of a $k$-cube. Combinatorica, $2(1): 1-7,1982$.

[2] D. Aldous. Brownian excursions, critical random graphs and the multiplicative coalescent. The Annals of Probability, pages 812-854, 1997.

[3] N. Alon, I. Benjamini, and A. Stacey. Percolation on finite graphs and isoperimetric inequalities. The Annals of Probability, 32(3):1727-1745, 2004.

[4] B. Blaszczyszyn. Lecture notes on random geometric models - random graphs, point processes and stochastic geometry. Lecture notes, December 2017.

[5] B. Bollobás. The evolution of random graphs. Transactions of the American Mathematical Society, 286(1):257-274, 1984.

[6] B. Bollobás, Y. Kohayakawa, and T. Euczak. The evolution of random subgraphs of the cube. Random Structures \& Algorithms, 3(1):55-90, 1992.

[7] C. Borgs, J. T. Chayes, R. van der Hofstad, G. Slade, and J. Spencer. Random subgraphs of finite graphs: I. The scaling window under the triangle condition. Random Structures 85 Algorithms, 27(2):137-184, 2005.

[8] C. Borgs, J. T. Chayes, R. van der Hofstad, G. Slade, and J. Spencer. Random subgraphs of finite graphs: II. The lace expansion and the triangle condition. The Annals of Probability, 33(5):1886-1944, 2005.

[9] C. Borgs, J. T. Chayes, R. van der Hofstad, G. Slade, and J. Spencer. Random subgraphs of finite graphs: III. The phase transition for the n-cube. Combinatorica, 26(4):395-410, 2006.

[10] F. Chung, P. Horn, and L. Lu. Percolation in general graphs. Internet Mathematics, 6(3):331-347, 2009.

[11] F. R. K. Chung and P. Tetali. Isoperimetric inequalities for cartesian products of graphs. Combinatorics Probability and Computing, 7(2):141-148, 1998.

[12] R. K. Chung. CBMS, spectral graph theory. American Mathematical Society, 1994.

[13] P. Erdős and A. Rényi. On random graphs I. Publ. math. Debrecen, 6(290-297):18, 1959. 
[14] P. Erdôs and A. Rényi. On the evolution of random graphs. Publ. Math. Inst. Hung. Acad. Sci, $5(1): 17-60,1960$.

[15] P. Erdôs and A. Rényi. On the strength of connectedness of a random graph. Acta Mathematica Academiae Scientiarum Hungarica, 12(1-2):261-267, 1964.

[16] P. Erdös and J. Spencer. Evolution of the n-cube. Computers \& Mathematics with Applications, 5(1):33-39, 1979.

[17] T. Hulshof and A. Nachmias. Slightly subcritical hypercube percolation. Random Structures 86 Algorithms, 56(2):557-593, 2020.

[18] S. Janson, T. Łuczak, and A. Rucinski. Random graphs, volume 45. John Wiley \& Sons, 2011.

[19] F. Joos. Random subgraphs in sparse graphs. SIAM Journal on Discrete Mathematics, 29(4):2350$2360,2015$.

[20] T. Łuczak. Component behavior near the critical point of the random graph process. Random Structures \&3 Algorithms, 1(3):287-310, 1990.

[21] C. McDiarmid. On the method of bounded differences. Surveys in combinatorics, 141(1):148-188, 1989.

[22] J.-P. Tillich. Edge isoperimetric inequalities for product graphs. Discrete Mathematics, 213(1-3):291320, 2000.

[23] R. van der Hofstad and A. Nachmias. Hypercube percolation. Journal of the European Mathematical Society, 19(3):725-814, 2017. 